Review Article

\title{
CHARACTERIZATION OF DRUGS ENCAPSULATED INTO MESOPOROUS SILICA
}

\author{
ARIF BUDIMAN \\ Department of Pharmaceutics and Pharmaceutical Technology, Faculty of Pharmacy, Universitas Padjadjaran, Indonesia \\ Email: arif.budiman@unpad.ac.id
}

Received: 11 Apr 2019, Revised and Accepted: 05 Oct 2019

\begin{abstract}
Solubility of the drug has a strong influence to achieve higher bioavailability of the drug in systemic circulation. More than 70\% NCEs (new chemical entities) are hydrophobic, and practically difficult into solid formulation due to their poor water solubility. Mesoporous silicas (MSP) have been used for drug delivery system, especially for poorly water-soluble drugs. Encapsulation and interaction of drugs in MSP can enhance the delivery and maintain the stability of the drug. However, the characterization of the drug in MSP is necessary to confirm its molecular state. In this review, we present an overview of reports related to the characterization of drug encapsulated into MSP. Encapsulation of drugs in MSP can prevent recrystallization of drugs due to its inhibition of crystal nucleation. A porous material in MSP can maintain the drug in a physically stable amorphous state. The preventing of drug crystallization in MSP can enhance the solubility and the dissolution rate of drug. Therefore, in this work, attempts have been made to understand the molecular state of the drug in MSP. The physicochemical characterization of drug by transmission electron microscopy (TEM), scanning electron microscope (SEM), differential scanning calorimetry (DSC), fourier-transform infrared spectroscopy (FTIR), powder x-ray diffraction (PXRD) and thermogravimetric analysis (TGA) were discussed. The effect of solvent and methods of drug loading and the effect of the shape of MSP on release profiles are also presented. Overall, this review provides information about the characterization of drug encapsulated into MSP which will be useful in pharmaceutical formulation development.
\end{abstract}

Keywords: Poorly water-soluble drug, Mesoporous silica, Amorphous, Dissolution enhancement

(c) 2019 The Authors. Published by Innovare Academic Sciences Pvt Ltd. This is an open-access article under the CC BY license (http://creativecommons.org/licenses/by/4.0/] DOI: http://dx.doi.org/10.22159/ijap.2019v11i6.35431

\section{INTRODUCTION}

The solubility of the drug has strong influence to achieve higher bioavailability of the drug in systemic circulation [1]. Base on the Bio-pharmaceutics Classification System (BCS), the drug in BCS Class II and Class IV are poor aqueous solubility [2]. More than 70\% NCEs (new chemical entities) are hydrophobic, and practically difficult into solid formulation due to their poor water solubility $[1,3]$. Developing strategies to modify the solubility of poorly watersoluble BCS class II and BCS class IV drugs is one of the greatest challenges in pharmaceutical research [4]

Some previous studies have been reported about the modification of drug solubility such as solid dispersions and nanoparticle which have led to enhance the dissolution of drugs [5]. However, the interactions between polymer and drug in solid dispersions is weak and cannot sufficiently stabilize the amorphous state of drug [6]. The aggregation of drugs nanoparticle can inhibit the dissolution enhancement [7]

In recent years, researchers have developed nanostructured materials for drug delivery. Mesoporous silica (MSP) has been used as a drug delivery system on the basis of silica-embedding and its biocompatibility. In MSP systems, drugs are adsorbed on the surface of silica and can affect the release rate of the drug in the precise sites of the body [8]. The drug delivery systems controlled by silica can improve therapeutic efficacy and reduce toxicity [9]. Mesoporous materials are good material for encapsulation of drug due to having a stable structure and well-defined surface properties [10]. The application of MSP has been widely reported as an oral drug delivery vehicles to increase the dissolution properties of the hydrophobic drug. Encapsulation and interaction of drugs in MSP can enhance the delivery and maintain the stability of the drug [11].

In this review, we present an overview of reports related to the characterization of drug encapsulated into MSP. Encapsulation of drug in MSP can prevent recrystallization of drugs due to its inhibition of crystal nucleation. A porous material in mesoporous silica can maintain the drug in amorphous state. The preventing of drug crystallization in MSP can enhance the solubility and the dissolution rate of drug $[12,13]$. Therefore, in this work, attempts have been made to understand the molecular state of the drug in MSP.
Physicochemical characterization of the drug inside mesoporous silica

\section{Differential scanning calorimetry (DSC) analysis}

The thermal behavior study of the drug in MSP usually used DSC. The purpose of using DSC measurement is to confirm that the drug can be incorporated inside MSP. The melting point data and glass transition data of the drug can clarify that mesoporous silica can prevent the recrystallization of the drug [14]. The heat of fusion of the endothermic peak of the drug will decrease along with the concentration of drug in MSP. Even, if all drugs already incorporated in MSP, the melting point of the drug did not exist. This indicated that the MSP has been successfully prevented the crystallization of drug [15]. The absence of a melting point or a glass transition related to monomolecular adsorption of drugs on the silica surface [14]. Table 1 presents the summary of a few kinds of literature reports the thermal behavior of drug in MSP.

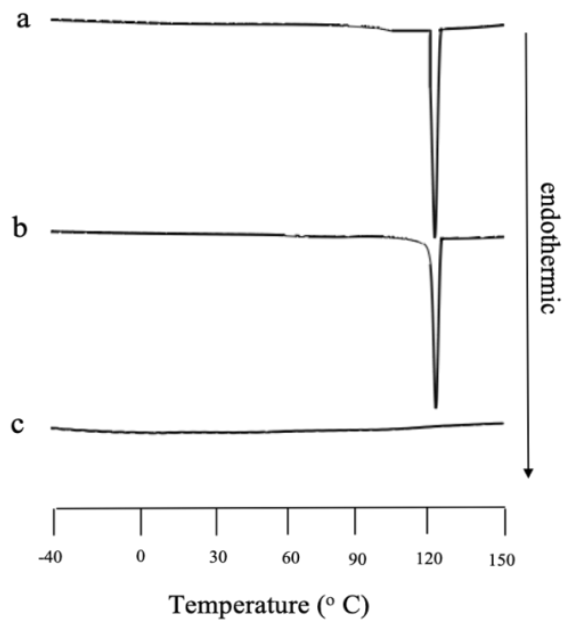

Fig. 1: The illustration of thermal behavior of (a) drug intact, (b) physical mixture of drug-MSP, (c) drug encapsulated into MSP 
Table 1: The summary of the thermal behavior analysis of drugs in MSP

\begin{tabular}{|c|c|c|c|c|c|c|}
\hline Name of API & $\begin{array}{l}\text { Therapeutics use for } \\
\text { API }\end{array}$ & $\begin{array}{l}\text { Mesoporous } \\
\text { silica type }\end{array}$ & $\begin{array}{l}\text { Tm of pure } \\
\text { drug }\end{array}$ & $\begin{array}{l}\text { Tm of drug in } \\
\text { mesoporous silica }\end{array}$ & $\begin{array}{l}\text { Comment on thermal } \\
\text { behaviour }\end{array}$ & References \\
\hline Ibuprofen & $\begin{array}{l}\text { Nonsteroidal anti- } \\
\text { inflammatory drug }\end{array}$ & SBA-15 & $78^{\circ} \mathrm{C}$ & - & $\begin{array}{l}\text { Successfully prevented the } \\
\text { crystallization of drug }\end{array}$ & 5,15 \\
\hline Itraconazole & Antifungal & $\begin{array}{l}\text { Neusilin UFL2 } \\
\text { SBA } 15 \\
\text { Ordered } \\
\text { mesoporous } \\
\text { silica (OMS) } \\
\text { Syloid AL-1 } \\
\text { and } 244\end{array}$ & $168^{\circ} \mathrm{C}$ & - & $\begin{array}{l}\text { Successfully prevented the } \\
\text { crystallization of drug }\end{array}$ & $\begin{array}{l}7 \\
5,17 \\
16 \\
18\end{array}$ \\
\hline Fenofibrate & $\begin{array}{l}\text { Treat abnormal blood } \\
\text { lipid levels }\end{array}$ & $\begin{array}{l}\text { SBA-15 and } \\
\text { MCM-41 }\end{array}$ & $81^{\circ} \mathrm{C}$ & - & $\begin{array}{l}\text { Successfully prevented the } \\
\text { crystallization of drug }\end{array}$ & 14 \\
\hline Glibenclamide & hypoglycaemic agent & SBA-15 & $170^{\circ} \mathrm{C}$ & - & $\begin{array}{l}\text { Monomolecular adsorption of } \\
\text { the drugs onto the surface of } \\
\text { SBA-15 }\end{array}$ & 19 \\
\hline Indhometachin & $\begin{array}{l}\text { Nonsteroidal anti- } \\
\text { inflammatory drug } \\
\text { (NSAID) }\end{array}$ & SBA-15 & $151.2^{\circ} \mathrm{C}$ & & $\begin{array}{l}\text { Monomolecular adsorption of } \\
\text { the drugs onto the surface of } \\
\text { SBA-15 }\end{array}$ & 19 \\
\hline Flurbiprofen & $\begin{array}{l}\text { Nonsteroidal anti- } \\
\text { inflammatory drug } \\
\text { (NSAID) }\end{array}$ & FSM-16 & $115.0^{\circ} \mathrm{C}$ & - & $\begin{array}{l}\text { FBP molecules adsorb into } \\
\text { the mesopores }\end{array}$ & 20 \\
\hline
\end{tabular}

Several studies mentioned that the endothermic peak of crystalline of drug will appear in the mesoporous system due to the high concentration of drug loading exceed the pore capacity. Nevertheless, the heat of fusion of drug is lower compared with pure drug [15]. The illustration of thermal behavior of drug in mesoporous silica can be seen in fig. 1 .

\section{Powder X-ray diffraction (PXRD) analysis}

The purpose of PXRD analysis is to investigate the molecular state of drug base on the diffraction peak of crystalline of drug. The limited size of mesoporous pore size prevent the formation of x-ray detectable crystalline of drug. If all drugs have been incorporated in
MSP, the drug in mesoporous system did not show the diffraction peak of crystalline of drug. Hardly any characteristic peaks were observed in the drug-loaded samples, suggesting that some drug loaded into MSP was in a nanocrystalline state [21]. Table 2 presents the summary of a few literature reports the PXRD analysis of drug in MSP.

Similar to DSC measurement, several studies mentioned that diffraction peak of drug will appear in the mesoporous system due to the high concentration of drug loading exceed the pore capacity. Nevertheless, the diffraction peak intensity of drug is lower compared with pure drug [15]. The illustration of diffraction peak of drug in MSP system can be seen in fig. 2 .

Table 2: The summary of PXRD analysis of drugs in MSP

\begin{tabular}{|c|c|c|c|c|c|}
\hline Drug & Mw (g/mol) & Carriers & Diffraction peak & $\begin{array}{l}\text { Comment on the } \\
\text { result }\end{array}$ & References \\
\hline Lovastatin & 404.5 & Mesoporous carbon & Almost undetectable & Nanocrystalline & 21 \\
\hline Curcumin & 368.38 & MCM-41 & displayed XRD pattern similar with MCM-41 & Nano-sized aggregates & 22 \\
\hline Telmisartan & 514.6 & $\begin{array}{l}\text { mesoporous silica } \\
\text { nanoparticles }\end{array}$ & no crystalline of drug & Noncrystalline state & 23 \\
\hline Itraconazole & 705.64 & Neusilin & Halo pattern & Amorphous state & 7 \\
\hline Piroxicam & 331.35 & MCM-41 & No crystalline peak was detected. & Noncrystalline state & 24 \\
\hline
\end{tabular}

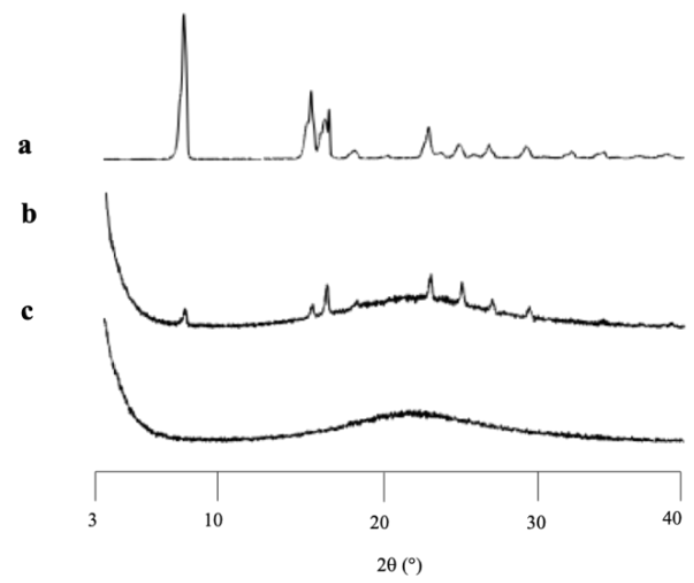

Fig. 2: The illustration of PXRD measurement of (a) drug intact, (b) drug encapsulated into MSP with excessive concentration, (c) drug encapsulated into MSP

\section{Fourier transformed infrared spectroscopy (FTIR) analysis}

The purpose of FTIR analysis is to observe the interaction of drug with MSP by the presence of functional group [25]. FTIR can provide the information about materials for identification of functional groups. Therefore, structural changes of drug and the lack of a crystal structure can be detected by FT-IR through the changing in bonding chemical groups of drug and MSP. The incorporation of drug on the mesoporous surface can be also qualitatively confirmed by FT-IR spectra [23].

The interaction of drug and MSP can be occurred physically and chemically. If the drug has interaction with MSP, the peak of drug will decrease or less sharp compared with pure drug spectrum [24]. The decrease of drug spectra in mesoporous system indicating the strong molecular interaction between drug and mesoporous, which would suggest to form salt. For physical interaction, the spectra of drug and MSP will appear, indicating the drug was adsorbed on the outer surface of the porous silica particles [26]. No structural change in the FTIR spectra also indicates that the drying process of the drug was sufficient to remove the solvent that used for incorporating drug into mesoporous [18]. 
The example case of FTIR measurement is an evaluation of ibuprofen loaded into MSP. The FTIR spectra of ibuprofen exhibited a single characteristic peak at $1711 \mathrm{~cm}^{-1}$ and three characteristic peaks between 2800 and $3300 \mathrm{~cm}^{-1}$. MSP A exhibited a stronger intensity band from 1300 to $900 \mathrm{~cm}^{-1}$. IBU-MSP A exhibited the combined individual characteristic peaks of IBU and MSP A, indicating that Ibuprofen was physically adsorbed on the surface of MSP. In contrast with IBU-MSP B, the characteristic peak of Ibuprofen showed at $1711 \mathrm{~cm}^{-1}$ was decreased to $1591 \mathrm{~cm}^{-1}$, indicating the strong intermolecular interactions between ibuprofen and MSP, which would suggest forming a salt [26]

\section{Morphology analysis}

The morphology and particle size of MSP were analyzed by SEM and TEM. The purpose of this study is to confirm the shape of MSP and investigate the nanoparticle structure of silica. However, the ability of SEM to verify the porous in detail is limited. Therefore, TEM is used to confirm the porous of silica [23]. The technique of Polarized light microscopy (PLM) also can be used to confirm the morphology of drug and MSP [26]. To investigate the morphology of the loading drug in MSP, the different shape can be used to confirm the morphology of drug in MSP using SEM, TEM and LPM.

The morphology of drug incorporated into MSP is not different with pure mesoporous. If the loading of drug increase, some new particles at surface of mesoporous will be observed $[7,15,23]$. This result indicated that the amount of drug exceeds the capacity of MSP. The uniform distribution of drug in MSP can be detected by TEM. The parallel stacked silica pore wall structure is well preserved can prove the uniform distribution of drug in MSP [15]. The PLM also shows similar result with the SEM result. If some bright spots were found in the images of drug-MSP, indicating that some drug was adsorbed on the outer surface of mesoporous. In contrast, if there is no significant difference between drug-MSP and pure MSP indicating all drug already loaded in MSP [26].

\section{Thermogravimetric analysis (TGA) analysis}

The purpose of TGA analysis in the characterization of drug encapsulated into MSP is to quantify the amount of drug encapsulated into mesoporous silica. The illustration of TGA analysis can be seen in fig. 3 .

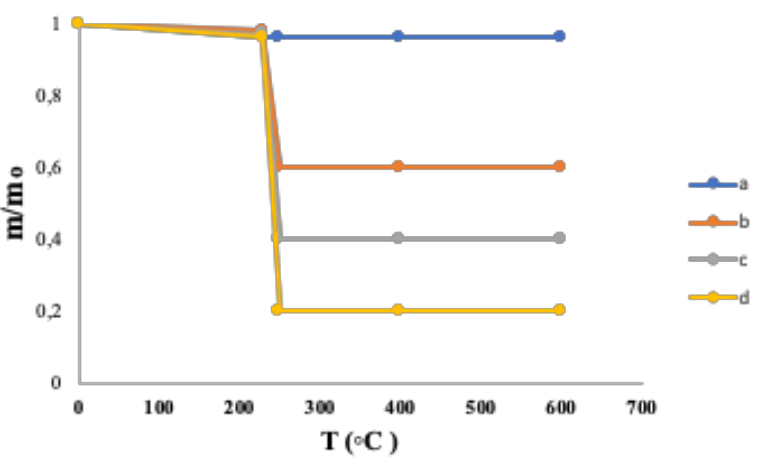

Fig. 3: The illustration of TGA analysis of (a) MSP, (b) MSP functionalized with aminopropyl group (c) washed sample, (d) unwashed sample

In the fig. 3, in MSP curve revealed two decreases. The first one is from room temperature until $100{ }^{\circ} \mathrm{C}$ indicated the mass loss and dehydration process of water adsorbed on the MSP. The second decrease is from 100 to $1000{ }^{\circ} \mathrm{C}$ indicated gradual condensation of the surface silanol groups, described as the process of dihydroxylation. For the line of $b$, the curve decrease indicating the weight loss of functional groups. Some article mentioned about the washed sample and unwashed sample. The purpose of this treatment is to quantify the drug only inside MSP. The result showed that the mass loss of the unwashed sample is higher than the washed sample. It is assumed that in the unwashed sample the drug not only inside MSP but also in outside of mesoporous silica. Or in the unwashed sample, the formation of the drug inside mesoporous silica can be achieved until two or three layers, while in the washed sample only a single monolayer [27-29].

\section{Nitrogen adsorption and desorption}

The specific area of MSP can be estimated by the nitrogen adsorption/desorption isotherms of typical type IV according to the IUPAC classification. The value of BET specific surface area (SBET), the total pore volume $(\mathrm{Vt})$ and the pore diameter $(\mathrm{dBJH})$ of MSP can be seen in table 3 .

Table 3: The total pore volume (Vt) and the pore diameter (dBJH) of MSP

\begin{tabular}{|c|c|c|c|c|c|c|c|c|}
\hline \multirow[t]{2}{*}{ Materials } & \multirow[t]{2}{*}{ drug } & \multicolumn{2}{|c|}{ SBet $\left(\mathrm{m}^{2} / \mathrm{g}\right)$} & \multicolumn{2}{|c|}{$V t\left(\mathrm{~cm}^{3} / \mathrm{g}\right)$} & \multicolumn{2}{|c|}{ dbjh (nm) } & \multirow[t]{2}{*}{ References } \\
\hline & & B & A & B & $\mathbf{A}$ & B & $\mathbf{A}$ & \\
\hline MSN & telmisartan & 585 & 527 & 1.51 & 1.16 & 12.9 & 10.4 & 23 \\
\hline SBA-15 & Itraconazole & 461 & 436 & 0.82 & 0.71 & 8.4 & 6.9 & 5 \\
\hline SBA-15 & Ibuprofen & 461 & 335 & 0.82 & 0.51 & 8.4 & 6.3 & 5 \\
\hline MCM-41 & Fenofibrate & 1028 & - & 0.73 & - & 2.7 & - & 14 \\
\hline MCM-41 & Lidocaine & 1553 & 827 & 1.75 & 1.63 & 2.61 & 1.75 & 30 \\
\hline MSN & Indomethacin & 1012 & 852 & 0.81 & 0.60 & 3.8 & 3.6 & 27 \\
\hline SBA-15 & ibuprofen & 734 & 307 & 1.05 & 0.57 & 6.0 & 5.6 & 15 \\
\hline
\end{tabular}

$\mathrm{B}=$ Before encapsulation, $\mathrm{A}=$ After Encapsulation

Drug loaded were characterized using nitrogen adsorption and desorption to investigate how the drug altered the porosity of MSP. The change of porosity upon loading drugs indicated that some drug had little influence on the porosity. The strong reduction of surface area, volume and pore diameter revealed some drug already inside MSP. The loading procedures, the interaction between drug-MSP and the solvent can effect the loading process of drug into MSP and can be characterized by nitrogen adsorption and desorption method [5].

\section{Effect of solvent selection on drug loading}

The solvent can affect the success of loading drug into MSP. The high solubility of the drug in the solvent can achieve a high concentration of drug-loaded into MSP [31-33]. However, the previous article reported that the solvent with the highest API solubility is not necessarily the best candidate for maximizing the API loading due to the competition between solvent and drug in the adsorption process. For the example valsartan with dichloromethane as the solvent can be incorporated into MSP 2x higher than methanol even though the concentration of valsartan in methanol was higher than in dichloromethane. Similarly, aprepitant loaded into MSP using chloroform as a solvent achieved more than $3 \mathrm{x}$ higher loading than methanol, even though the concentration of aprepitant in methanol was $3 \mathrm{x}$ higher than chloroform [34].

To explore about the solvent effect systematically, Soltys, et al. conducted the experiment with the same drug (ibuprofen) and the same concentration $(10 \mathrm{mg} / \mathrm{ml})$ that was dissolved in six different solvent to incorporate the drug into MSP. The result of soltys et al. experiment can be seen in table 4 . 
Table 4: The loading of ibuprofen into mesoporous silica at $10 \mathrm{mg} / \mathrm{ml}$ in different solvents [34]

\begin{tabular}{lll}
\hline Solvents & Ibuprofen loading (wAPI/w Si \%) & Solvent dielectric constant \\
\hline Ethanol & 3.5 & 24.5 \\
Isopropanol & 3.9 & 18.0 \\
Methanol & 4.3 & 33.0 \\
Acetone & 4.8 & 21.0 \\
Chloroform & 6.4 & 4.8 \\
Dichloromethane & 8.8 & 9.1 \\
\hline
\end{tabular}

Base on the table 4, the best performance of loading ibuprofen into MSP was dichloromethane. Chloroform is slightly lower than dichloromethane but has a higher solubility which can compensate for the lower efficiency. Ethanol and isopropanol showed poorly result with the loading below $4 \%$. While methanol and acetone are slightly better with the loading of $4.3 \%$ and $4.8 \%$ respectively [34]. In this result, we can conclude that the solvent with a lower dielectric constant generally performed better compared with the higher dielectric constant. This result also similar to the work of Stam et al. and makilla et al., which showed the poor performance of methanol and better performance of chloroform [35, 36].

\section{Effect of methods on drug loading}

The understanding of the state of drug adsorbed at the surface of MSP is very important. This related to the loading procedures of drug incorporated into MSP. Mellaerts et al. reported about the comparison of loading procedures of the drug (itraconazole) into MSP using three different loading methods, viz, solvent evaporation method, incipient wetness impregnation, and melting method [5, 37]. The result of Mallaert et al. work about itraconazole loading can be seen in table 5 [5].

Base on the table 5, the result showed that using the solvent method either solvent evaporation method or incipient wetness impregnation revealed a good agreement between drug loadings according to TGA and release under sink conditions. When using melt method, larger discrepancies were observed. The thermal degradation of itraconazole might be occurred from heat treatment. The high viscosity of drug after melted can affect the flow of liquid of drug into MSP and prevent a homogenous distribution [5].

Table 5: The result of itroconazole loading into MSP with various methods

\begin{tabular}{lll}
\hline Formula & TGA & Sink release \\
\hline Solvent-10 \% & 6.09 & 6.19 \\
Solvent-30 \% & 27.88 & 26.55 \\
Impregnation-10\% & 6.75 & 5.44 \\
Impregnation-30 \% & 29.53 & 27.32 \\
Melt-10\% & 7.02 & 5.15 \\
Melt-30\% & 24.7 & 22.74 \\
\hline
\end{tabular}

Another method that can be used to incorporate the drug into MSP is co-spray drying. Shen et al. have been conducted the incorporation of drug (ibuprofen) into MSP using co-spray drying and compared with ball milling, impregnation and melt method. The result showed that base on PXRD, in weight ratio 50:50 (drug: MSP) using the cospray drying method did not show any diffraction peak of the crystalline form of ibuprofen. Similar to PXRD result, in DSC measurement also did not show the endothermic peak of ibuprofen. It is assumed that in ratio 50:50 using co-spray drying, ibuprofen has successfully incorporated into MSP.

In contrast with ball milling, impregnation and melt method, the result of PXRD measurement of this method showed that in ratio 50:50 still revealed the crystalline peak of ibuprofen. In DSC measurement, the ratio 50:50 using those methods also showed strong endothermic peak of ibuprofen. It is assumed that in ratio 50:50 using ball milling, impregnation and melt method, some ibuprofen still in outside the mesoporous silica. The co-spray drying is more efficient compared with ball milling, impregnation and melt method due to the rapid drying of atomized solvent can prevent the formation of ibuprofen crystal [15].

\section{Effect of shape on physicochemical properties of MSP}

Many scientists have synthesized MSP to improve the bioavailability of poorly water-soluble drugs with varying size, surface charge, and morphology of MSP [38]. Shen et al. reported that the pore size and particle size of MSP can influence the dissolution profile of ibuprofen. To investigate the systematic studies about the effect of shape on the dissolution test of poorly-water soluble, Zhang et al. conducted the synthesis of MSP with different shapes (spherical and rod) and investigated the behavior of MSP in their drug delivery properties [39].

The result showed that the surface area of the rod-shaped MSP rodshaped was 1.3 fold higher than the sphere-shaped. Alkyl alcohol was important for controlling the morphologies of MSP. Alcohol can decrease the critical micelle concentration triggering the formation of rod-shaped MSP [39]. Based on PXRD measurement, the absence of diffraction peaks indicated that the rod-shaped and the sphere-shaped of MSP were in the amorphous state [40]. In the dissolution test, the drug incorporated into rod-shaped MSP was faster than the sphere-shaped. Similar to the dissolution test, in the in vivo study showed that the rod-shaped 2.2 fold higher than the sphere-shaped. This indicated that the different shape of MSP can affect the behaviour of drugs incorporated into MSP [41].

\section{CONCLUSION}

MSP is a promising method to increase the solubility of poorly water-soluble drugs. Encapsulation of drugs in MSP can prevent recrystallization of drugs due to its inhibition of crystal nucleation. A porous material in MSP can maintain the drug in a physically stable amorphous state. The preventing drug crystallization in MSP can enhance the solubility and the dissolution rate of drug. Investigation of drug encapsulated into mesoporous silica is very important to understand the molecular state of drug in MSP. The method and the solvent can affect the success of loading drug into MSP. The shape of MSP also can affect drug delivery properties.

\section{ACKNOWLEDGMENT}

None

\section{ABBREVIATIONS USED}

NCEs: new chemical entities; MSP: Mesoporous silica; TEM: transmission electron microscopy; SEM: scanning electron microscope; DSC: differential scanning calorimetry; FTIR: Fouriertransform infrared spectroscopy; PXRD: powder x-ray diffraction; TGA: thermo gravimetric analysis; BCS: Bio-pharmaceutics Classification System; Tm: melting point; Tg: glass transition

\section{AUTHORS CONTRIBUTIONS}

All the author have contributed equally

\section{CONFLICT OF INTERESTS}

No conflict of interest to declare 


\section{REFERENCES}

1. Skorupska E, Jeziorna A, Potrzebowski MJ. Thermal solventfree method of loading of pharmaceutical cocrystals into the pores of silica particles: a case of naproxen/picolinamide cocrystal. J Phys Chem C 2016;120:13169-80.

2. Edward KH, Li D. Drug Like Properties: Concept, Structure, Design and Methods, from ADME to Toxicity Optimization; Solubility Elsevier: New York, NY, USA; 2008. p. 56.

3. Sathisaran I, Dalvi S. Engineering cocrystals of poorly watersoluble drugs to enhance dissolution in an aqueous medium. Pharmaceutics 2018;10:108.

4. Skorupska E, Paluch P, Jeziorna A, Potrzebowski MJ. NMR study of BA/FBA cocrystal confined within mesoporous silica nanoparticles employing thermal solid phase transformation. J Phys Chem C 2015;119:8652-61.

5. Mellaerts R, Jammaer JA, Van Speybroeck M, Chen H, Humbeeck JV, Augustijns P, et al. Physical state of poorly water-soluble therapeutic molecules loaded into SBA-15 ordered mesoporous silica carriers: a case study with itraconazole and ibuprofen. Langmuir 2008;24:8651-9.

6. Yamamoto K, Kojima T, Karashima M, Ikeda Y. Physicochemical evaluation and developability assessment of co-amorphouses of low soluble drugs and comparison to the co-crystals. Chem Pharm Bull 2016;64:1739-46.

7. Azad M, Moreno J, Dave R. Stable and fast-dissolving amorphous drug composites preparation via impregnation of Neusilin® UFL2. J Pharm Sci 2018;107:170-82.

8. Wang S. Ordered mesoporous materials for drug delivery. Micropor Mesopor Mat 2009;117:1-9.

9. Wang Y, Zhao Q, Han N, Bai L, Li J, Liu J, et al. Mesoporous silica nanoparticles in drug delivery and biomedical applications. Nanomed Nanotechnol 2015;11:313-27.

10. Slowing II, Trewyn BG, Giri S, Lin VY. Mesoporous silica nanoparticles for drug delivery and biosensing applications. Adv Func Mat 2007;17:1225-36.

11. Kinnari P, Makila E, Heikkila T, Salonen J, Hirvonen J, Santos HA. Comparison of mesoporous silicon and non-ordered mesoporous silica materials as drug carriers for itraconazole. Int J Pharm 2011;414:148-56.

12. Ali KH, Ansari MM, Shah FA, Din FU, Basit MA, Kim JK, et al. Enhanced dissolution of valsartan-vanillin binary coamorphous system loaded in mesoporous silica particles. J Microencapsul 2019;36:1-11.

13. Bi Y, Xiao D, Ren S, Bi S, Wang J, Li F. The binary system of ibuprofen-nicotinamide under nanoscale confinement: from cocrystal to coamorphous state. J Pharm Sci 2017;106:3150-5.

14. Van Speybroeck M, Mellaerts R, Mols R, Do Thi T, Martens JA, Van Humbeeck J, et al. Enhanced absorption of the poorly soluble drug fenofibrate by tuning its release rate from ordered mesoporous silica. Eur J Pharm Sci 2010;41:623-30.

15. Shen SC, Ng WK, Chia L, Dong YC, Tan RB. Stabilized amorphous state of ibuprofen by co-spray drying with mesoporous SBA-15 to enhance dissolution properties. J Pharm Sci 2010;99:1997-2007.

16. Mellaerts R, Mols R, Jammaer JA, Aerts CA, Annaert P, Van Humbeeck J, et al. Increasing the oral bioavailability of the poorly water-soluble drug itraconazole with ordered mesoporous silica. Eur J Pharm Biopharm 2008;69:223-30.

17. Mellaerts R, Aerts CA, Van Humbeeck J, Augustijns P, Van den Mooter G, Martens JA. Enhanced release of itraconazole from ordered mesoporous SBA-15 silica materials. Chem Comm 2007;13:1375-7

18. Kinnari P, Makila E, Heikkila T, Salonen J, Hirvonen J, Santos HA. Comparison of mesoporous silicon and non-ordered mesoporous silica materials as drug carriers for itraconazole. Int J Pharm 2011;414:148-56.

19. Van Speybroeck M, Mellaerts R, Thi TD, Martens JA, Van Humbeeck J, Annaert P, et al. Preventing release in the acidic environment of the stomach via occlusion in ordered mesoporous silica enhances the absorption of poorly soluble weakly acidic drugs. J Pharm Sci 2011;100:4864-76.

20. Tozuka Y, Wongmekiat A, Kimura K, Moribe K, Yamamura S, Yamamoto K. Effect of pore size of FSM-16 on the entrapment of flurbiprofen in mesoporous structures. Chem Pharm Bull 2005;53:974-7.
21. Zhao P, Wang L, Sun C, Jiang T, Zhang J, Zhang Q, et al. Uniform mesoporous carbon as a carrier for poorly water soluble drug and its cytotoxicity study. Eur J Pharm Biopharm 2012;80:535-43.

22. Jambhrunkar S, Karmakar S, Popat A, Yu M, Yu C. Mesoporous silica nanoparticles enhance the cytotoxicity of curcumin. RSC Adv 2014;4:709-12.

23. Zhang Y, Zhi Z, Jiang T, Zhang J, Wang Z, Wang S. Spherical mesoporous silica nanoparticles for loading and release of the poorly water-soluble drug telmisartan. J Controlled Release 2010;145:257-63.

24. Ambrogi V, Perioli L, Marmottini F, Giovagnoli S, Esposito M, Rossi C. Improvement of dissolution rate of piroxicam by inclusion into MCM-41 mesoporous silicate. Eur J Pharm Sci 2007;32:216-22.

25. Xu W, Riikonen J, Lehto VP. Mesoporous systems for poorly soluble drugs. Int J Pharm 2013;453:181-97.

26. Lai J, Lin W, Scholes P, Li M. Investigating the effects of loading factors on the in vitro pharmaceutical performance of mesoporous materials as drug carriers for ibuprofen. Materials 2017;10:150.

27. Hu L, Sun C, Song A, Chang D, Zheng X, Gao Y, et al. Alginate encapsulated mesoporous silica nanospheres as a sustained drug delivery system for the poorly water-soluble drug indomethacin. Asian J Pharm Sci 2014;9:183-90.

28. Kiwilsza A, Milanowski B, Drużbicki K, Coy LE, Grzeszkowiak M, Jarek $\mathrm{M}$, et al. Mesoporous drug carrier systems for an enhanced delivery rate of poorly water-soluble drug: nimodipine. J Porous Mat 2015;22:817-29.

29. Meka A, Jenkins L, Davalos Salas M, Pujara N, Wong K, Kumeria $\mathrm{T}$, et al. Enhanced solubility, permeability and anticancer activity of vorinostat using tailored mesoporous silica nanoparticles. Pharmaceutics 2018;10:283.

30. Nafisi S, Samadi N, Houshiar M, Maibach HI. Mesoporous silica nanoparticles for enhanced lidocaine skin delivery. Int J Pharm 2018;550:325-32.

31. Heikkila T, Salonen J, Tuura J, Kumar N, Salmi T, Murzin DY, et al. Evaluation of mesoporous TCPSi, MCM-41, SBA-15, and TUD-1 materials as API carriers for oral drug delivery. Drug Delivery 2007;14:337-47.

32. Hong S, Shen S, Tan DCT, Ng WK, Liu X, Chia LS, et al. High drug load, stable, manufacturable and bioavailable fenofibrate formulations in mesoporous silica: a comparison of spray drying versus solvent impregnation methods. Drug Delivery 2016;23:316-27.

33. Salonen J, Laitinen L, Kaukonen AM, Tuura J, Bjorkqvist M, Heikkila T, et al. Mesoporous silicon microparticles for ora drug delivery: loading and release of five model drugs. J Controlled Release 2005;108:362-74.

34. Soltys M, Kovacik P, Dammer O, Beranek J, Stepanek F. Effect of solvent selection on drug loading and amorphization in mesoporous silica particles. Int J Pharm 2019;555:19-27.

35. Makila E, Kivela H, Shrestha N, Correia A, Kaasalainen M, Kukk $\mathrm{E}$, et al. Influence of surface chemistry on ibuprofen adsorption and confinement in mesoporous silicon microparticles. Langmuir 2016;32:13020-9.

36. Hillerstrom A, Andersson M, Samuelsson J, van Stam J. Solvent strategies for loading and release in mesoporous silica. Colloid Interfac Sci 2014;3:5-8.

37. Sanjay C, Ghate VM, Lewis SA. Mesoporous silica particles for dermal drug delivery: a review. Int J Appl Pharm 2018;10:23-6.

38. Banerjee A, Qi JP, Gogoi R, Wong J, Mitragotri S. Role of nanoparticle size, shape, and surface chemistry in oral drug delivery. J Controlled Release 2016;238:176-85.

39. Landry CC, Tolbert SH, Gallis KW, Monnier A, Stucky GD, Norby $\mathrm{F}$, et al. Phase transformations in mesostructured silica/surfactant composites. Mechanisms for change and applications to materials synthesis. Chem Mat 2001;13:1600-8.

40. Sharmiladevi S, Priya AS, Sujitha M. Synthesis of mesoporous silica nanoparticles and drug loading for gram-positive and gram-negative bacteria International. J Pharm Pharm Sci 2016;8:196-201.

41. Zhang W, Zheng N, Chen L, Xie L, Cui M, Li S, et al. Effect of shape on mesoporous silica nanoparticles for oral delivery of indomethacin. Pharmaceutics 2019;11:4. 